\title{
Escleritomos de Chancelloria eros y escleritos del Cámbrico medio (Serie 3, Piso 5) de Sonora central, México
}

\author{
Matilde S. Beresi ${ }^{1, *}$, Blanca Buitrón-Sánchez ${ }^{2,{ }^{* *}}$, Francisco J. Cuen-Romero ${ }^{3}$, Juan J. Palafox ${ }^{3}$ \\ ${ }^{1}$ Instituto Argentino de Nivología, Glaciología y Ciencias Ambientales (IANIGLA), Consejo Nacional de Investigaciones \\ Científicas y Tecnológicas (CONICET), Av. A. Ruiz Leal s/n, Parque General San Martín, 5500, Mendoza, Argentina. \\ ${ }^{2}$ Universidad Nacional Autónoma de México, Instituto de Geología. Departamento de Paleontología, \\ Ciudad Universitaria, Ciudad de México, C.P. 04510, México. \\ ${ }^{3}$ Universidad de Sonora, División de Ciencias Exactas y Naturales, \\ Departamento de Geología, Blvd. Luis Encinas y Rosales, Hermosillo, Sonora, C.P. 83000, México. \\ *mberesi@mendoza-conicet.gob.ar,**blancab@unam.mx
}

\section{RESUMEN}

Fragmentos de escleritomos de Chancelloria eros del Cámbrico medio de Sonora, México se registran por primera vez. Escleritomos de chancellóridos parcialmente articulados y escleritos aislados pertenecientes a Allonnia tintinopsis y Archiasterella sp. se encuentran ampliamente distribuidos en calcilutitas rojizas de la Formación El Gavilán, en la sección del Sahuaral, Sonora central. La fauna está compuesta por chancellóridos, braquiópodos linguliformes, espículas de esponjas reticulosas, trilobites y hyolítidos. La Formación El Gavilán es una secuencia de plataforma abierta de $65 \mathrm{~m}$ de espesor, compuesta de calcilutita intercalada con caliza margosa de estratificación delgada. La asociación de trilobites permitió asignar el depósito al Cámbrico medio, Subzona de Altiocculus, Zona de Ehmaniella (Serie 3, Piso 5). Los géneros de chancellóridos de la sección de El Sahuaral pueden ser comparados con la biota de San José de Gracia, Sonora central, México y también con depósitos cámbricos de la plataforma alrededor de Laurentia como son Burgess Shale en Canadá y formaciones de Utah en EUA. Las rocas del Cámbrico de Sonora fueron parte de la periferia suroeste del cratón de Laurentia, la cual se ubicaba cerca del paleo-ecuador durante el Cámbrico.

Palabras clave: Chancelloria eros; Allonnia; Archiasterella; Altiocculus Subzona; Cámbrico medio; Sonora; México.

\section{ABSTRACT}

Fragments of scleritomes of Chancelloria eros from the middle Cambrian of Sonora, Mexico are reported for the first time. Chancelloriid scleritomes partially articulated and isolated sclerites belonging to Allonnia tintinopsis and Archiasterella sp. are widely distributed in reddish calcilutite of the El Gavilán Formation, in the Sahuaral section, central Sonora. The faunal association is represented by chancelloriids, spicules of reticulosan sponges, linguliform brachiopods, hyoliths and trilobites. The El Gavilán Formation is an open shelf sequence of $65 \mathrm{~m}$ thick, composed of calcilutite interbedded with thin-bedded marly limestone. Trilobite association allowed assigning the open shelf deposit to the middle Cambrian, Altiocculus Subzone, Ehmaniella Zone (Series 3, Stage 5).
Chancelloriid genera of the El Sahuaral section may be compared with the biota of San Jose de Gracia, in central Sonora, México, and also with other mid-Cambrian shelf deposits around Laurentia as Burgess Shale in Canada and formations from Utah, USA. Cambrian rocks of Sonora were part of the southern-western border of Laurentia craton, which was located near the paleo-equator during Cambrian times.

Key words: Chancelloria eros; Allonnia; Archiasterella; Altiocculus Subzone; middle Cambrian; Sonora; Mexico.

\section{INTRODUCCIÓN}

La distribución geográfica de las rocas sedimentarias del Cámbrico en México es limitada, localizándose la mayoría en los estados de Sonora, Chihuahua y Oaxaca en afloramientos aislados y esporádicos (Buitrón, 1992; Cuen, 2012). El registro estratigráfico del Cámbrico en Sonora se caracteriza por rocas clásticas y carbonáticas de ambiente marino somero, distribuidas en diferentes afloramientos en la parte norte, centro y este del estado. Estos afloramientos poseen una diversa fauna fósil estudiada desde el siglo pasado por Lochman (1948) y Cooper et al., (1952, 1956), entre otros. La columna bioestratigráfica del Cámbrico se encuentra basada principalmente en los alcances estratigráficos de trilobites, debido a su abundancia y amplia distribución geográfica. En Sonora, han sido objeto de numerosos estudios paleontológicos y sistemáticos (Lochman en Cooper et al., 1952; González-León, 1986; Almazán-Vázquez, 1989).

Entre los metazoos cámbricos compuestos por espinas, los chancellóridos representan un grupo enigmático que fue originariamente asignado al Phylum Porífera por Walcott, 1920 y posteriormente interpretados como pertenecientes al grupo de los coeloscleritóforos (Bengtson y Missarzhevsky, 1981) los que florecieron y se expandieron durante la "Explosión Cámbrica" asociados a shelly faunas. Estratigráficamente se encuentran restringidos a rocas del Cámbrico temprano y medio tanto en Laurentia (faunas de Groenlandia, de Burgess Shale (Walcott, 1920) y Utah (Rigby, 1978), como en Australia (Mehl, 1998), China (Qian y Bengtson, 1989) y en la Precordillera Argentina (Beresi y Rigby 1994, 2013). Los tres géneros de chancellóridos Chancelloria, Allonnia y Archiasterella tienen registros desde el Cámbrico temprano en el

Beresi, M.S., Buitrón-Sánchez, B., Cuen-Romero, F.J., Palafox, J.J., 2019, Escleritomos de Chancelloria eros y escleritos del Cámbrico medio (Serie 3, Piso 5) de Sonora central, México: Revista Mexicana de Ciencias Geológicas, v. 36, núm. 1, p. 54-63. 
Sur de China, en la plataforma de Yangtzé (Qian y Bengtson, 1989). Hacia el Cámbrico tardío el grupo muestra una rápida declinación y se extinguen antes de finales del Cámbrico.

Los chancellóridos son organismos sésiles que vivieron adheridos al sustrato o a otros organismos, y de posibles hábitos de alimentación por filtración. Tienen un cuerpo en forma de saco alargado, con apariencia de cactus, denominado escleritomo, cubierto por espinas llamadas escleritos que se insertan en el tegumento. Los escleritos en forma de roseta, son huecos y compuestos, de simetría radial. El grupo es conocido principalmente por escleritos aislados, desarticulados, de amplia distribución en plataformas del Cámbrico inferior y medio. Sin embargo, la preservación de escleritomos completos articulados o parcialmente articulados es excepcional. Tanto su posición filogenética como su tratamiento taxonómico han sido motivo de controvertidos análisis (p.e. Moore et al., 2014).

En el Cámbrico de México, los chancellóridos son conocidos exclusivamente por escleritos disociados. Los primeros estudios de chancellóridos en México corresponden a los realizados en el área de Caborca, Sonora por Lochman (in Cooper et al., 1952) para la Formación Arrojos del Cámbrico medio, quien menciona la presencia de escleritos de Chancelloria eros Walcott, 1920, en asociación con otros taxa. McMenamin (2008) reporta la existencia de Chancelloria sp. en asociación con Kiwetinokia ? para la Formación Puerto Blanco (Zona de Nevadella) en Cerro Rajón y San Clemente, en Caborca. Mientras que para Sonora central, en el área de San José de Gracia el primer registro de escleritos de Chancelloria eros y Allonnia asociados a espículas de esponja, braquiópodos y trilobites representativos del Cámbrico medio (Zona de Mexicella mexicana), corresponde a Cuen et al. (2013). Posteriormente, Beresi et al. (2013) describen una asociación de escleritos de chancellóridos y espículas de esponjas hexactinéllidas para la misma localidad de Sonora central.

En el Cerro El Sahuaral se han realizados escasos estudios estratigráficos y paleontológicos. Stewart y Poole (2002) mencionan los trilobites Peronopsis sp., Bathyuriscus sp. y Oryctocephalus sp. que permiten asignar estas rocas al Cámbrico medio. Recientemente, Cuen-Romero et al. (2018) describen la fauna de trilobites de localidades típicas del Cámbrico de Sonora.

En el presente trabajo se describen por primera vez para el Cámbrico de México, fragmentos de escleritomos (cuerpos de chancellóridos) parcialmente articulados de Chancelloria eros Walcott, 1920 y escleritos de Allonnia tintinopsis Bengtson y Collins, 2015. Escleritos parcialmente articulados asignados a Archiasterella sp. (y con dudas a A. charma Moore et al., 2014) se describen e ilustran para la nueva localidad de estudio.

\section{LOCALIZACIÓN DEL ÁREA DE ESTUDIO}

El material estudiado procede del Cerro El Sahuaral, con coordenadas geográficas $29^{\circ} 19.540^{\prime} \mathrm{N}$ y $110^{\circ} 35.834^{\prime} \mathrm{W}$, ubicado a $5 \mathrm{~km}$ al noreste del ejido de San José de Gracia, en la parte central del estado de Sonora (Figura 1). El Cerro El Sahuaral se localiza a $44 \mathrm{~km}$ al noroeste de Hermosillo, siendo esta la ciudad más importante próxima al área de estudio. El Cerro El Sahuaral forma una prominencia aislada con elevación de 370 m s.n.m.

\section{MARCO ESTRATIGRÁFICO}

En el área de estudio la secuencia estratigráfica del Paleozoico inferior está representada de base a techo por las formaciones Proveedora, Buelna, Cerro Prieto y El Gavilán. En discordancia erosiva se apoyan depósitos volcánicos del Mioceno, así como aluvión y coaluvión del Holoceno. En la base, se han observado secciones transversales de arqueociátidos en posición de vida inmersos en caliza gris claro, lo que permite inferir el registro de rocas equivalentes a la Formación Puerto Blanco definida en Caborca por Cooper et al., (1952) por primera vez para Sonora central en la sección de estudio.

La fauna de chancellóridos aquí estudiada fue recolectada en la Formación El Gavilán una secuencia de plataforma constituida por

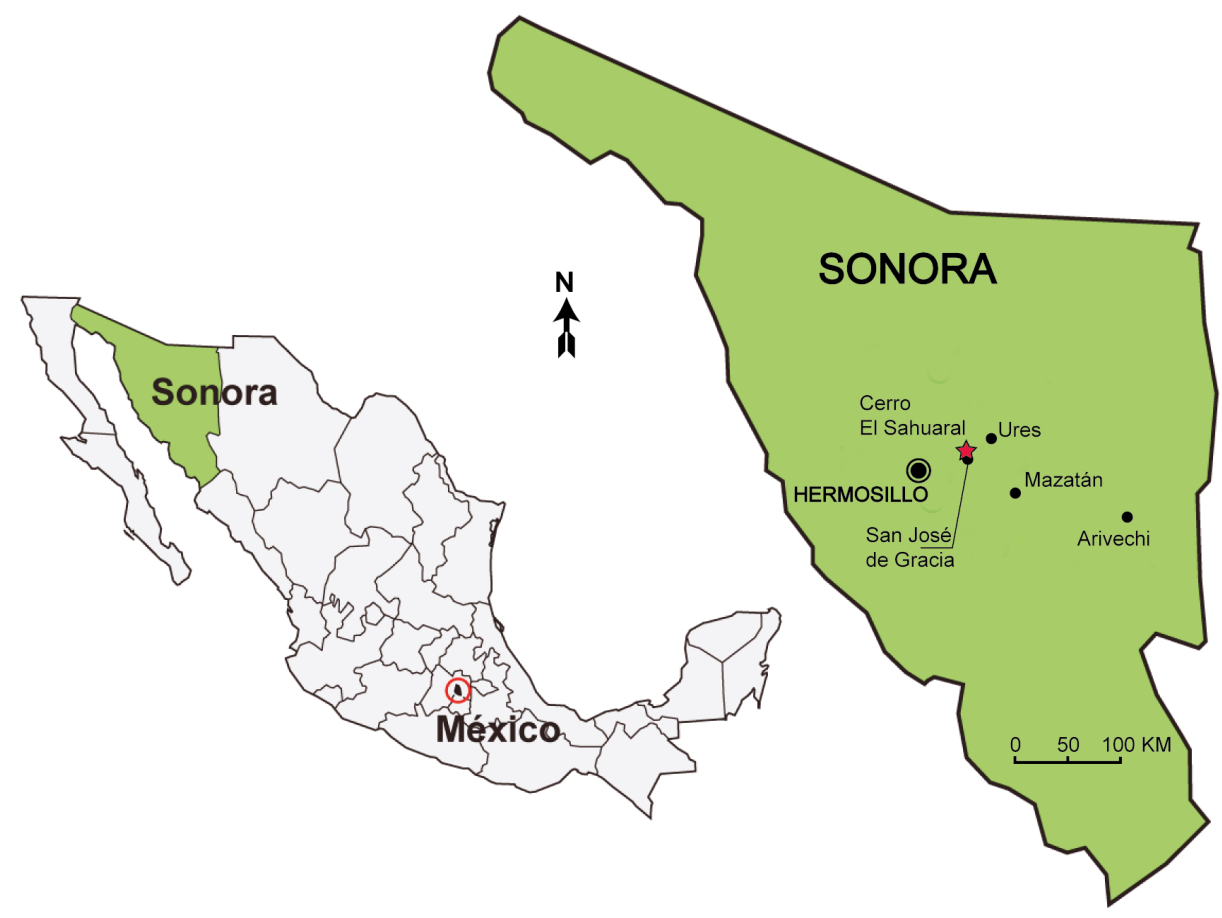

Figura 1. Mapa de Sonora, México y localización del área de estudio. 
intercalaciones mayoritarias de calcilutita de tonalidades rojizas con caliza delgada aflorante en la sección estratigráfica de El Sahuaral. Esta formación fue definida en el área del cerro El Chihuarruita, San José de Gracia, su sección tipo, con un espesor de $233 \mathrm{~m}$ y por la asociación de trilobites fue asignada a la parte superior de la Zona de Mexicella mexicana, Subzona de Albertella highlandensis, Zona de Ptychagnostus praecurrens (Cuen-Romero et al., 2016). La sección tipo de la Formación El Gavilán en San José de Gracias, se encuentra localizada a 7 km de la sección El Sahuaral, en Sonora central (Figura 2).

La fauna fósil de la sección cámbrica de El Sahuaral, es diversa y moderadamente abundante. El mayor porcentaje corresponde a trilobites ptychopariidos y braquiópodos linguliformes (Acrothele sp., Prototreta sp.), fragmentos de escleritomos (Chancelloria eros) y escleritos de chancellóridos aislados (Allonnia y Archiasterella), espículas de esponjas hexactinéllidas, (Protospongia sp. y Diagoniella sp.) y en menor proporción moluscos (hyolítidos) y restos indeterminados. La asociación de trilobites es representativa de la Subzona de Altiocculus, parte superior de la Zona de Ehmaniella (Sundberg, 1994) y en consecuencia los depósitos portadores de la Formación El Gavilán tienen una edad correspondiente a la Serie 3 (Piso 5) y al Linconiano (Delamarano) de Laurentia (Cuen-Romero et al., 2018).

La depositación de estas rocas corresponde a un ambiente de plataforma abierta, de aguas cálidas, que bordeaba el continente de Laurentia durante el Cámbrico temprano y medio. La región suroeste de este continente correspondiente a Sonora actual se localizaba cerca del paleoecuador.

\section{PALEONTOLOGÍA SISTEMÁTICA}

El material estudiado e ilustrado en esta publicación está depositado en la colección de referencia del Departamento de Geología de la Universidad de Sonora, México, bajo los números de catálogo USDG 581- 593.

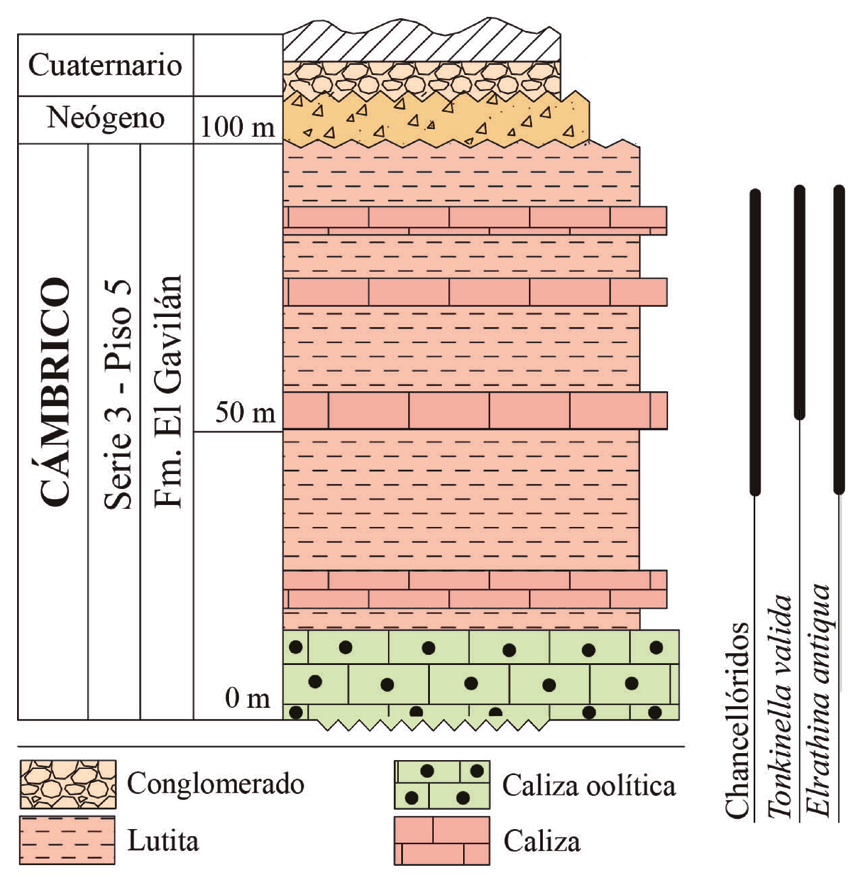

Figura 2. Perfil estratigráfico de la Formación El Gavilán en la sección de El Sahuaral, Sonora central, México.
Coeloscleritophora Bengtson y Missarzhevsky, 1981

Orden Chancelloriida Walcott, 1920

Familia Chancelloriidae Walcott, 1920

Género Chancelloria Walcott, 1920

Especie tipo. Chancelloria eros Walcott, 1920; Cámbrico medio; Burgess Shale, Columbia Británica, Canadá.

\section{Chancelloria eros Walcott, 1920}

Figuras 3a, 3b, 3c, .3d, 3e, 3f, 3g y 4b, 4e

Rigby, 1978, p.1330, fig. 3; p. 1342, fig. 5; Beresi y Rigby, 1994, p. 215, figs. 5-9, p. 216, fig. 6; Zhao et al., 1999, p.11, fig 7; Janussen et al, 2002 , p. 599 , fig. 3 , p. 600 , fig. 4, p. 602 , fig. 6.1 ; Bengtson, 2000 , p. 11-12, figs. 12-13; Bengtson y Collins, 2015, p. 11-17, figs. 5-11, p. 19-20, figs. 13-14.

Descripción. Sobre el plano de estratificación de una calcilutita de tonalidades rojizas de $2.50 \mathrm{~cm}$ de espesor se observa un agrupamiento natural de escleritos (USDG-581) que representan parte del cuerpo de un individuo (escleritomo) parcialmente articulado de la especie Chancelloria eros Walcott, 1920. El escleritomo tiene forma aproximadamente cilíndrica de $17 \mathrm{~mm}$ de longitud y un diámetro máximo de 9-10 mm (Figura 3a, 3b). El conjunto parece haber sido algo desplazado de su posición original, debido a que la orientación apicalabapical (basal) difiere entre los escleritos. Los escleritos presentan la típica forma estrellada con un disco central desde el cual los rayos se articulan tangencialmente. En general se puede trazar un plano medial que divide al esclerito en dos partes simétricas. Predominan los escleritos de fórmula $6+1$ y algunos escasos de $7+1$, sin mayores variaciones en tamaño y longitud de los rayos. La mayoría de los escleritos están articulados y reemplazados por óxidos de hierro. Los escleritos se observan en relieve positivo y negativo correspondientes a las caras opuestas del escleritomo que ha sido aplastado. El disco basal es de forma poligonal con un diámetro promedio de 0.20-035 mm, alcanzando hasta $0.45 \mathrm{~mm}$. Los rayos marginales tienen entre $0.55-0.75 \mathrm{~mm}$ de longitud. Las facetas basales de articulación de los rayos con el disco central son de aproximadamente $0.10 \mathrm{~mm}$. De entre los rayos tangenciales uno puede ser notoriamente curvo con longitudes entre $0.8 \mathrm{~mm}$ y $2.0 \mathrm{~mm}$ mientras que los otros rayos marginales varían en longitud entre $0.50-0.75$ hasta $1.2 \mathrm{~mm}$. El nodo central que corresponde a la parte basal del rayo central no preservado tiene un diámetro de $0.15 \mathrm{~mm}$ (Figura $4 \mathrm{~b}$ ). Algunos escleritos tienen algunos rayos marginales de similar longitud entre de 0.50 y $0.75 \mathrm{~mm}$ y otros más largos y algo curvos. La separación entre dos escleritos varía entre 1.0 y $1.2 \mathrm{~mm}$. Sobre la cara inferior de algunos escleritos se observa el foramen circular (orificio) en la base de cada rayo a través del cual se conectaban con el tejido epidermal del cuerpo del organismo.

En otra calcilutita de tonalidades rojizas de $3.4 \mathrm{~cm}$ de espesor (USDG-582) numerosos escleritos están naturalmente agrupados, distribuidos paralelos al plano de estratificación. Los conjuntos de escleritos corresponden a fragmentos de escleritomos parcialmente articulados (Figura 3e, 3f, 3g). Se distinguen como parches de formas irregulares más o menos alargados, de una tonalidad rojiza más intensa que la matriz, sin bordes delineados y esparcidos sobre el plano de la roca. Los parches tienen entre 4 y $5 \mathrm{~cm}$ por $2 \mathrm{~cm}$ en la parte más ancha. No se observa una clara regularidad en la distribución de los escleritos. Los escleritos están articulados y en general bien preservados en relieve tanto positivo como negativo, con un relleno de óxido de hierro (posiblemente hematita), destacándose muy bien sobre la matriz de tonalidad rojiza más pálida. Exhiben predominantemente la fórmula de rayos 7+1 y menos de 6+1, con un disco basal de 0.35-0.45 mm. Los rayos marginales son en general rectos con algún rayo que se curva 

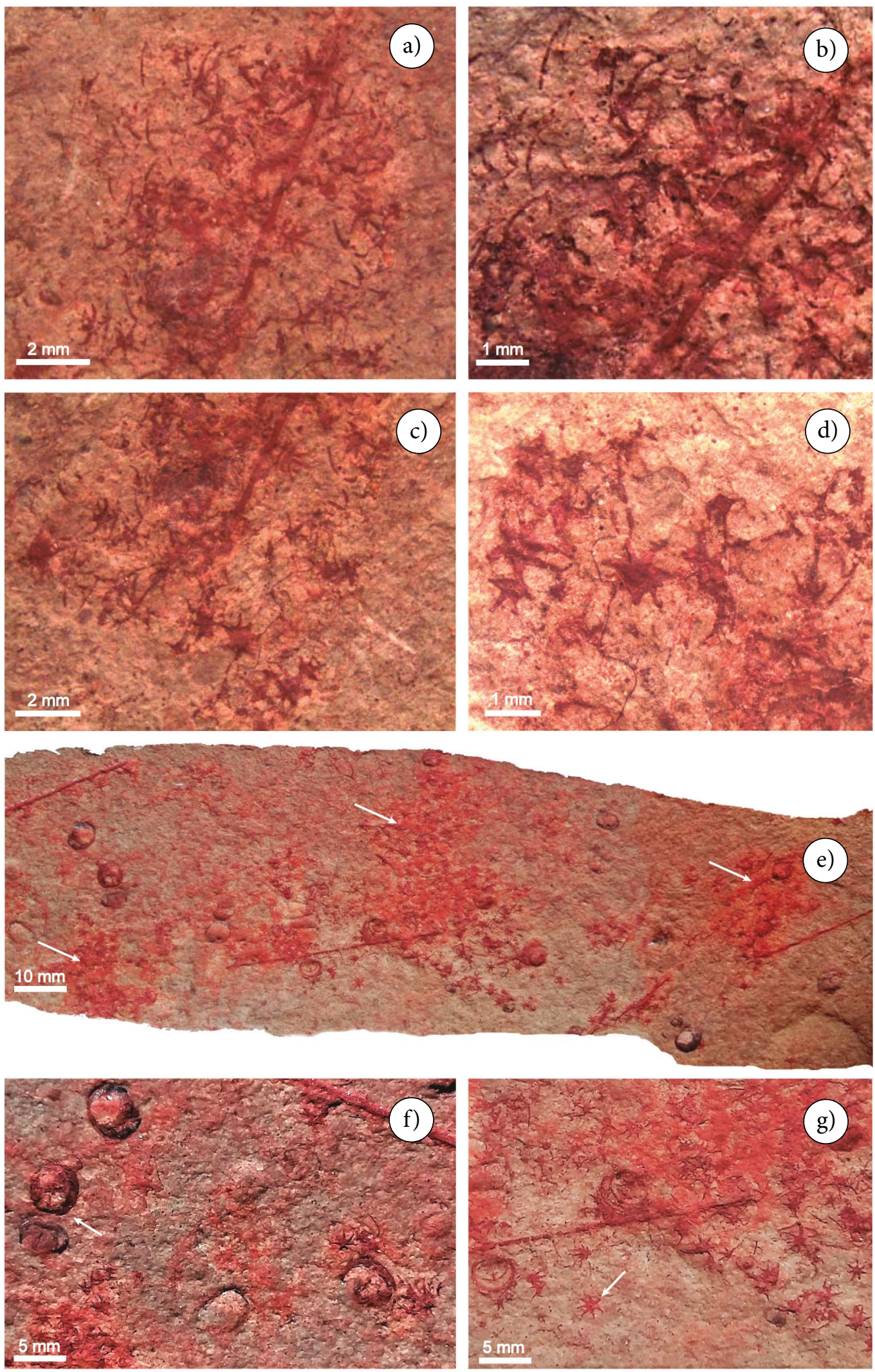

Figura 3. Escleritomos de Chancelloria eros Walcott, 1920. a) Vista general del escleritomo USON 581; b) vista de la parte superior; c) vista de la parte media; d) detalle de escleritos; e) fragmentos de escleritomos de C. eros (flechas), USON 582; f) y g) detalle de escleritomo de C. eros; f) braquiópodos linguloideos, posibles epibiontes (flecha); g) braquiópodos linguloideos y escleritos con fórmula 7+1 (flecha). 
posiblemente hacia la parte distal (apical) del cuerpo del chancellórido. La longitud de los rayos varía entre $0.9-1.2 \mathrm{~mm}$. En un esclerito el tamaño de los rayos marginales puede ser variable como la base de articulación con el disco central.

En algunos agrupamientos de mayor tamaño se observa una cierta dirección predominante de los rayos de mayor longitud que, comparados con Chancelloria eros (Bengtson y Collins, 2015), posiblemente apunten hacia el orificio distal (ápice) del organismo.

Escasos escleritos aislados del género Allonnia, como también valvas desarticuladas de braquiópodos linguliformes de aproximadamente 2-5 mm de longitud se distribuyen entre los escleritos.

Sobre los planos de estratificación de varias calcilutitas rojizas de 2-3 cm de espesor (USDG 583-587) se observan escleritos aislados (Figura 4a, 4c, 4d, 4f) la mayoría articulados y otros con rayos fragmentados o no preservados. Predominan escleritos de $7+1$ rayos pero con $6+1$ rayos son comunes. Los rayos de mayor tamaño tienen longitudes de 0.75 hasta $1.7 \mathrm{~mm}$ desde la base de inserción con el disco central. Algunos rayos marginales se curvan distalmente con longitudes entre 1.2 y $1.7 \mathrm{~mm}$ mientras que los otros son rectos y progresivamente se adelgazan hacia el extremo con longitudes variables entre $0.50-1.5 \mathrm{~mm}$. En algunos escleritos se observa la protuberancia correspondiente a la base del rayo central vertical no preservado. La articulación basal entre los rayos y el disco central varía entre 0.35 y $0.50 \mathrm{~mm}$. En sección basal se observa el foramen circular localizado en la parte proximal de los rayos.

Distribución. Cámbrico medio, Serie 3, Piso 5, Subzona de Altiocculus parte superior de la Zona de Ehmaniella ( 505 Ma).

Material. Fragmentos de escleritomos sobre el plano de estratificación de dos calcilutitas de la Formación El Gavilán, Cerro El Sahuaral, Sonora, México. Números de catálogo USDG-581y 582. Calcilutitas con numerosos escleritos aislados (USDG 583-587).

Discusión. Las características morfológicas de los escleritos analizados de las muestras USDG-581y 582 con fórmula dominante de 6-7+1 rayos y con tamaño del disco central de 0.25 a $0.45 \mathrm{~mm}$ permiten asignar estos fragmentos de escleritomos a la especie Chancelloria eros Walcott, 1920. En los agrupamientos de escleritos de la muestra USG 582, la forma de los escleritomos no está delineada por lo que no es posible determinar el número de individuos o si pertenecen a un mismo organismo o a diferentes. Esos parches contienen numerosos escleritos de morfología y fórmula de rayos idénticos por lo que se infiere que podrían corresponder a relictos de varios escleritomos de la misma especie. En el material analizado de Sonora no se han observado escleritos de fórmula 3-4+0 que son mencionados como raros, muy escasos componentes en las descripciones de C. eros (Walcott, 1920, Bengtson y Collins, 2015).

Por las características morfológicas y fórmula de rayos $(6-7+1)$ de los escleritos correspondientes a fragmentos de escleritomos y de los escleritos aislados aquí descritos se infiere que pertenecen a la especie Chancelloria eros Walcott, 1920 descrita entre otros por Mehl (1996), Janussen et al. (2002) para Chengjiang, China y Wheeler Shale, EUA y por Bengtson y Collins (2015) para el yacimiento clásico de Burgess Shale, en Canadá.

Género Allonnia Doré y Reid, 1965

Especie tipo. Allonnia tripodophora Doré y Reid, 1965; Cámbrico inferior, Carteret, Macizo Armoricano, Francia.

Allonnia tintinopsis Bengtson y Collins, 2015

Figuras $4 \mathrm{f}$ y $5 \mathrm{a}, 5 \mathrm{~b}, 5 \mathrm{e}, 5 \mathrm{f}$

Bengtson y Collins, 2015, p. 7-8, figs. 2-3; 12 (part); p. 23-38, figs.17-30;
31.1-31.3; 32; p. 43, fig. 37

Descripción. Sobre el plano de calcilutitas se observan diversos escleritos aislados y articulados, con fórmula $3+0$. Se caracterizan por dos largos y delgados rayos curvos en forma de lira, con longitudes de 0.60- $1.55 \mathrm{~mm}$, alcanzando hasta $3.7 \mathrm{~mm}$, con un promedio de $1.3 \mathrm{~mm}$ y ancho basal de los rayos de 0.12 a $0.20 \mathrm{~mm}$. Los dos rayos laterales forman un ángulo aproximado de $70^{\circ}$. El tercer rayo ascendente no se observa debido a que los escleritos están aplastados sobre el plano de la roca.

Distribución. Cámbrico medio, Serie 3, Piso 5, Subzona de Altiocculus parte superior de la Zona de Ehmaniella ( 505 Ma).

Material. Escleritos de chancellóridos en calcilutitas rojizas de la Formación El Gavilán, Cerro El Sahuaral, Sonora, México; número de catálogo USDG 587-588-591.

Discusión. La morfología de los escleritos de Sonora y el tamaño de los rayos son muy similar a los de la especie Allonia tintinopsis (Bengtson y Collins, 2015). La especie Al. phrixothrix Bengtson y Hou (2001) tiene rayos de mayor longitud $(8 \mathrm{~mm}) \mathrm{y}$ ancho basal de los rayos $(0.4 \mathrm{~mm})$. En la especie Al. erjiensis Yun et al. (2017) la forma del esclerito es diferente por tener rayos triangulares y más robustos que se adelgazan rápidamente hacia el ápice formando un ángulo agudo entre sí. Además, la longitud y el ancho basal de los rayos varía de acuerdo a la posición que ocupan en el escleritomo con rayos entre 1.5 hasta $3.8 \mathrm{~mm}$ de longitud y ancho basal entre 0.3 y $0.4 \mathrm{~mm}$. Al. tintinopsis fue descrita por Bengtson y Collins (2015) para las lutitas de Burgess Shale, en Canadá, siendo la especie de chancellóridos más común dentro de esa fauna cámbrica.

Género Archiasterella Sdzuy, 1969

Especie tipo. Archiasterella pentactina Sdzuy, 1969; Cambrico inferior (Atdabanian), Cazalla de la Sierra, Ossa Morena, España; basado en asociación de escleritos

\section{Archiasterella sp. \\ Figura $5 c-5 d$}

Sdzuy, 1969, p. 134, fig.10; Qian and Bengtson, 1989, p. 18, fig. 6a-c; Fernández-Remolar, 2001, p. 58, fig. 8c-d, f, i-l; Beresi y Rigby, 2013, p.271, fig. $8 \mathrm{~g}-\mathrm{k}$.

Descripción. Dos escleritos desarticulados y aislados con fórmula de rayos 3+0 presentan el rayo ascendente (basal de Randell et al., 2005 o principal de Moore et al., 2014.) muy bien desarrollado de $4.5 \mathrm{~mm}$ (Figura 5c) y $6.5 \mathrm{~mm}$ (Figura 5d) de longitud y robustos en su parte proximal, con 0.9 y $1.1 \mathrm{~mm}$ de diámetro respectivamente. El rayo curva distalmente y perpendicularmente a los dos rayos horizontales que están reducidos a una longitud de $1.5 \mathrm{~mm}$ en el esclerito de la Figura $5 \mathrm{~d}$. La superficie planar-adapical de estos rayos es casi horizontal y mide $2.0 \mathrm{~mm}$ de ancho. En el esclerito de la Figura $5 \mathrm{c}$ se ha preservado sólo el rayo ascendente que curva distalmente y los rayos laterales están completamente reducidos.

Distribución. Cámbrico medio, Serie 3 Piso 5, Subzona de Altiocculus parte superior de la Zona de Ehmaniella.

Material. Dos escleritos aislados en calcilutitas rojizas de la Formación El Gavilán, sección El Sahuaral, Sonora; números de catálogo USDG 589-590.

Discusión. La especie tipo de Archiasterella Sdzuy, 1969, tiene un escleritomo dominado por fórmula $5+0$. Por esta razón, en la literatura prevaleció la tendencia de asignar aquellos escleritos de 5+0 al género Archiasterella y todos aquellos de $3+0$ al género Allonnia.

De acuerdo con el criterio de Moore et al., (2014) que se sigue en este trabajo, se considera que el arreglo de los rayos con respecto a la 
base de inserción es una característica determinante para la asignación taxonómica de estos géneros, más que el número de rayos que puede ser variable en un mismo individuo, siendo éste el criterio que ha sido preferentemente seguido en la literatura del grupo. En los archiasteréllidos la base de inserción es plana a diferencia del género Allonnia cuyos rayos curvan desde la base hacia el ápice formando un ángulo mayor a $45^{\circ}$. Otra característica de ambos géneros es la ausencia de un rayo central perpendicular a los rayos marginales como ocurre típicamente en Chancelloria.
En el caso de los escleritos que aquí se describen la morfología de su rayo vertical permiten una asignación sistemática al género Archiasterella teniendo como característica distintiva la base de inserción de los rayos horizontales casi plana (Figura 5d). Como el esclerito de Figura 5d, está desarticulado, se podría inferir que los dos rayos laterales y el rayo vertical se fragmentaron, y en este caso la fórmula de rayos sería 5+0, como por ejemplo en la especie Archiasterella fletchergryllus Randell et al., 2005, o A. pentactina Sdzuy, 1969. Otra interpretación posible es considerar que los escleritos fueron origina-
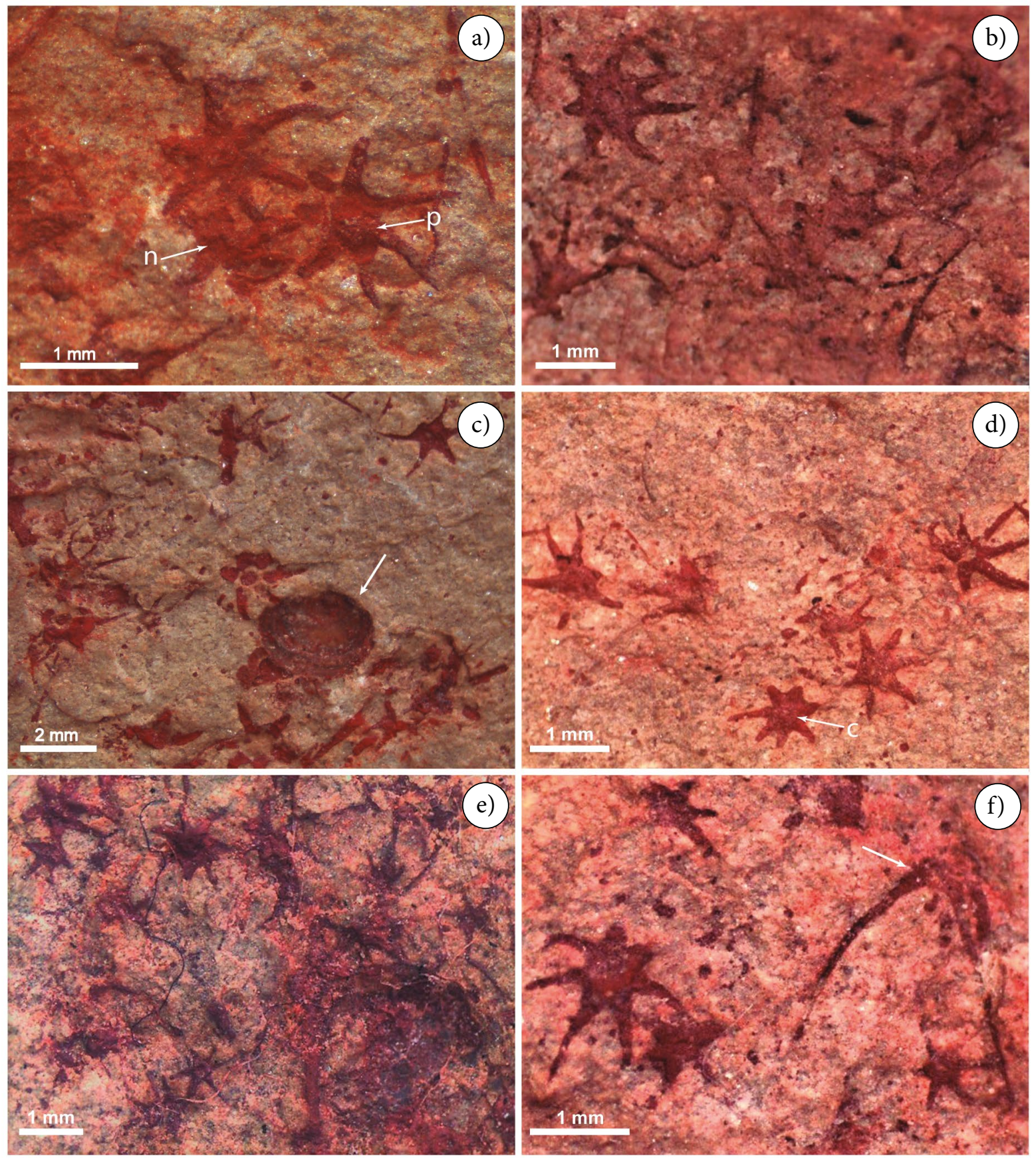

Figura 4. Escleritos aislados de Chancelloria eros Walcott, 1920. a) Escleritos en relieve positivo (p) y negativo (n) de fórmula de rayos 6-7+1, USON 583; b) USON 584; c) valva interior de braquiópodo linguliforme (flecha) y escleritos, USON 585; d) esclerito con rayo central (c), USON 586; e) detalle de escleritomo, USON 581; f) escleritos de C. eros y Allonnia tintinopsis (a), USON 587. 
riamente de 3+0 rayos como la especie Archiasterella charma (Moore et al., 2014) descrita para el Cámbrico inferior del este de Yunnan, China. El esclerito de la Figura 5d, es muy similar en forma y longitud del rayo principal (ascendente) a dicha especie. Entre otras especies cámbricas $A$. hirundo Bengtson, 1990 tiene $4+0$ rayos por lo que difiere de los escleritos de Sonora. En el esclerito ilustrado en la Figura $5 \mathrm{c}$ se ha preservado sólo el rayo ascendente y la base circular de los rayos laterales muy reducida. Debido a la disponibilidad de sólo dos escleritos en la colección de El Sahuaral con regular preservación no es posible una asignación sistemática específica.

\section{Fauna acompañante}

Figuras $3 e, 3 f, 3 g$ y $6 a$, $6 b$

La asociación biótica de la Formación El Gavilán en la sección de estudio está compuesta por chancellóridos, braquiópodos, hiolítidos
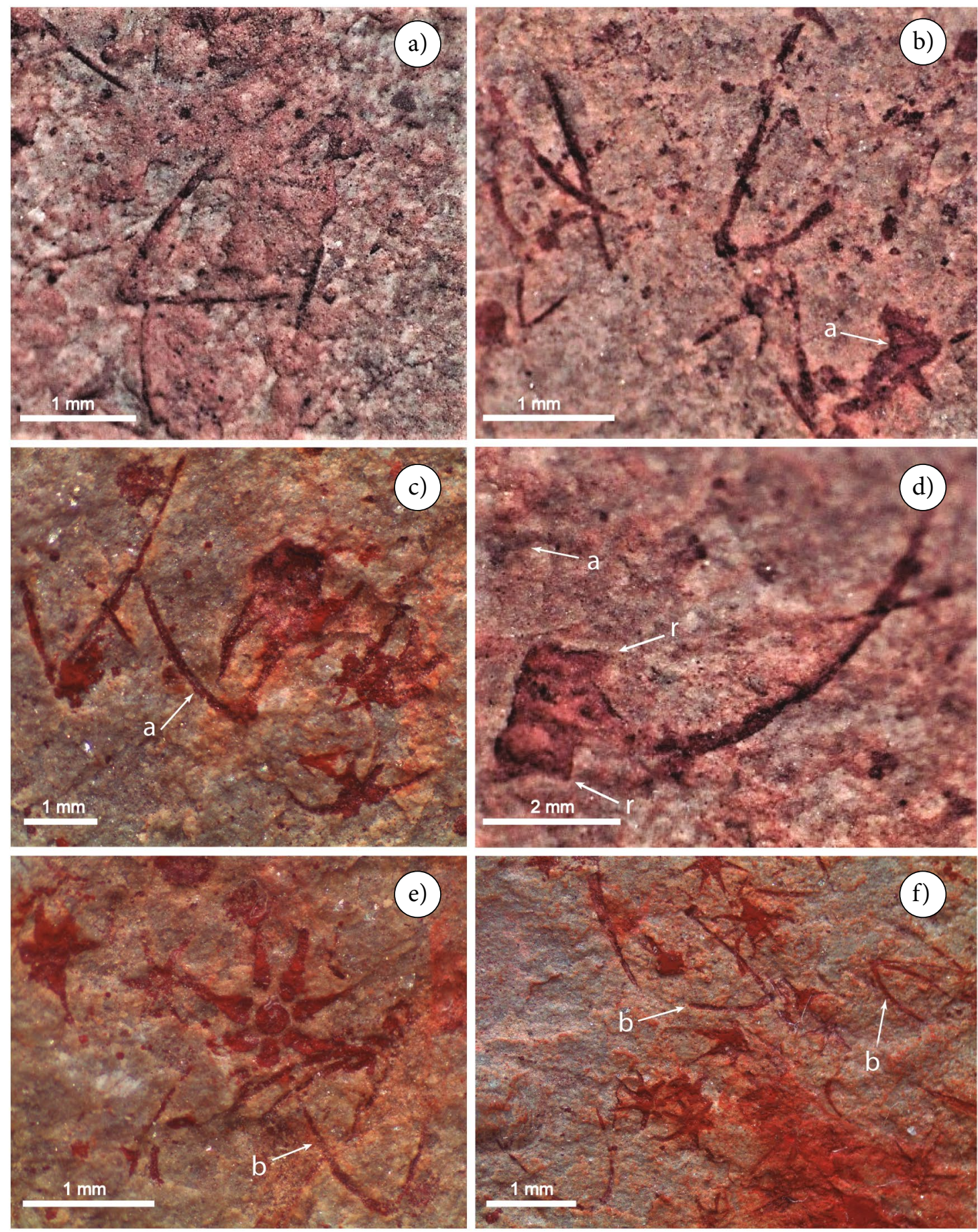

Figura 5. Chancelloriidae. a, b) Escleritos de Allonnia tintinopsis Bengtson y Collins, 2015, y de Archiasterella (a), USON 588; c) esclerito de Archiasterella sp. (a), USON 589; d) esclerito de Archiasterella sp. con los dos rayos horizontales reducidos (r), USON 590; e) y f) escleritos de Al. tintinopsis (b) y C. eros, USON 591. 
y trilobites. De manera particular, la presencia del trilobite Tonkinella valida, asociado a Quadragnostus sp. y Elrathina antiqua (Figura 6a, 6b) son indicadores de la parte superior de la Zona de Ehmaniella, Subzona de Altiocculus (Sundberg, 1994).

De manera general la presencia predominante de trilobites de la Familia Spinagnostidae (Quadragnostus) y Familia Oryctocephalidae (Tonkinella), indican condiciones ambientales de plataforma exterior. También es notorio la abundancia de braquiópodos linguliformes (Acrothele) y espículas estauractinas sueltas de esponjas reticulosas (hexactinéllidas). Sobre los parches de escleritos se observan valvas de braquiópodos linguliformes de tamaño predominante entre 2 a $5 \mathrm{~mm}$ de largo. Es posible interpretar que estos braquiópodos utilizaron como sustrato a los escleritomos de Chancelloria eros a modo de epibiontes (Figuras 3f, g y 4c). Por ejemplificar, especímenes del braquiópodo Acrothyra gregaria anexados a escleritos de Chancelloria eros fueron ilustrados por Bengtson y Collins (2015, figs. 9.2, 9.3) en ejemplares del yacimiento de Burgess Shale, Canadá.

En la sección de estudio entre la fauna de braquiópodos linguliformes se han identificado Dictyonina sp.y Acrothele sp. Mientras que en la misma formación pero en la sección tipo de San José de Gracia se determinaron además de los mencionados Prototreta sp., Linnarsonia sp. y Lingulella sp. (Cuen-Romero et al., 2016) y los hiolítidos Hyolithes sonora Lochman en Cooper et al., 1952 y Haphophrentis reesei Babcock y Robinson, 1988 (Buitrón et al., 2011).

De acuerdo a la biota analizada, los trilobites son predominantes, seguidos por braquiópodos linguliformes y en escasa proporción, espículas de esponjas y escleritos de chancellóridos. Sin embargo, en los niveles donde están presentes, los escleritos pueden ser abundantes y el resto de la fauna disminuye.

\section{CONSIDERACIONES BIOESTRATIGRÁFICAS}

La Formación El Gavilán del área del cerro El Sahuaral contiene ejemplares de Quadragnostus depressa, Tonkinella valida y Elrathina antiqua asociados a Chancelloria eros, Allonia tintinopsis y Archiasterella sp. La presencia de los trilobites mencionados anteriormente indica una edad del Cámbrico medio (Serie 3, Piso 5). El contenido biótico es asignado a la Zona de Ehmaniella, Subzona de Altiocculus (Figura 6).
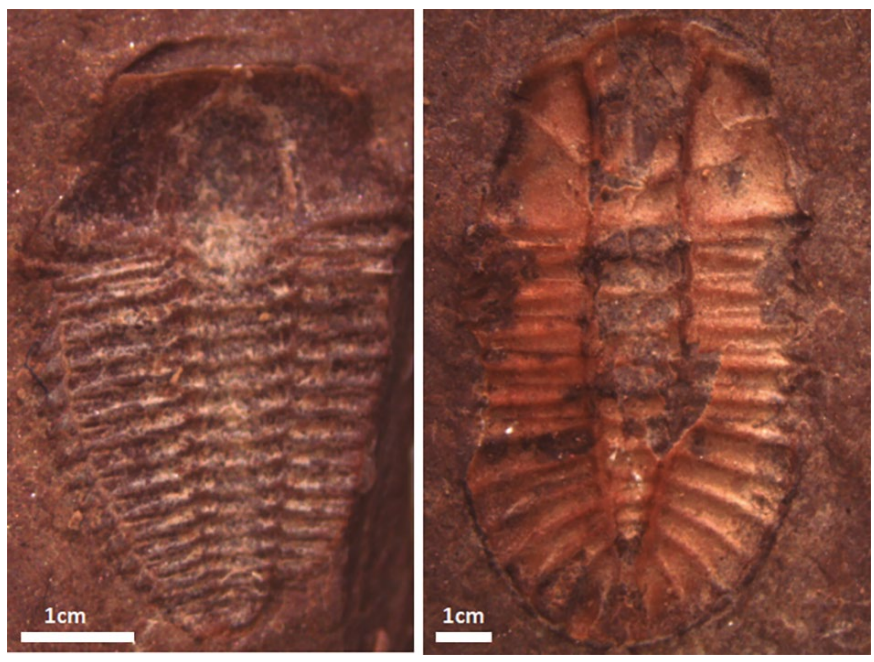

Figura 6. Trilobites de la Zona de Ehmaniella, Subzona de Altiocculus (Sundberg, 1994). a) Elrathina antiqua USON 592. b) Tonkinella valida USON 593.
La Zona de Ehmaniella fue definida por Sundberg (1994), como una biozona de conjunto que ocurre sobre la Zona de Glossopleura y debajo de la Zona de Bolaspidella (Cámbrico medio), dividiéndola en cuatro subzonas, de la base a la cima: Proehmaniella, Elrathiella, Ehamniella y Altiocculus. La Zona de Ehmaniella ha sido reconocida en el estado de Sonora por Vega-Granillo (1996) en Rancho Sobechi, Mazatán, con base en la presencia de los trilobites Quadragnostus depressa (=Peronopsis fallax), Tomagnostus sp., Tonkinella valida y Syspacephalus sp., siendo asignados a la Subzona de Altiocculus por Cuen (2013). Por lo tanto, el presente trabajo constituye el segundo registro de la Zona de Ehmaniella para México.

El contenido faunístico de chancellóridos de la Formación El Gavilán en la sección estratigráfica de El Sahuaral es prácticamente idéntico al de la misma formación en la sección tipo del Cerro El Chihuarruita en San José de Gracia. Entre los chancellóridos de ambas localidades se recolectaron escleritos sueltos de Chancelloria y Allonnia junto a espículas y fragmentos de tejido espicular de esponjas reticulosas (hexactinéllidas), braquiópodos linguloides como Acrothele sp. y Prototreta sp., además de la asociación de trilobites (completos o en fragmentos desarticulados de cranidios y tórax) junto con placas del eocrinoideo Gogia (Nardin et al., 2009) y hiolítidos (Buitrón et al., 2011).

Chancelloria, Allonnia y Archiasterella tienen registros desde el Cámbrico temprano en diversos sitios de la plataforma cámbrica de Laurentia, Gondwana y China. Desde el Cámbrico inferior y medio las faunas tipo Burgess Shale se distribuyeron especialmente alrededor del borde occidental de Laurentia (Figura 7), desde el norte de Groenlandia, en Sirius Passet (Conway-Morris et al., 1987), en los clásicos depósitos de Burgess Shale en la Columbia Británica (Walcott, 1920; Janussen et al., 2002) y en los territorios del Noroeste de Canadá (Randell et al., 2005); en la Formación Marjum y Weeler Shale de Utah, EUA (Rigby,1978), hasta Sonora (Cuen et al., 2013; Beresi et al., 2017). También estas faunas están presentes en el margen este de Laurentia, y en Gondwana (Fernández-Remolar, 2001), China (Zhang et al., 2008) y Corea (Hong et al. 2016). Incluyen diversos y numerosos taxa, entre los que predominan artrópodos (no trilobites), esponjas, chancellóridos, hyolítidos, moluscos, eocrinoideos y algas entre otros.

Escleritomos parciales o completos de Chancelloria eros del Cámbrico inferior y medio se han descrito en las lutitas de Burgess Shale (Walcott, 1920; Bengtson y Collins, 2015), en la Formación Wheeler y Marjum de Utah, EUA (Rigby, 1978; Janussen et al., 2002), en la Formación Kaili, China (Zhao et al., 1999) y en la Precordillera Argentina (Beresi y Rigby, 2013) entre otros. En las lutitas de Burgess Shale, Cámbrico medio (Drumiano), Bengtson y Collins, (2015) describen escleritomos articulados de Allonnia tintinopsis, mientras que la especie Archiasterella charma Moore et al., (2014) fue descrita para del Cámbrico inferior (pre-trilobites) basada exclusivamente en escleritos disociados en el este de la Provincia de Yunnan, China.

La asignación de escleritos de Sonora a la especie Allonnia tintinopsis constituye hasta el presente la segunda mención de esta especie a nivel global y la descripción de escleritomos incompletos de Chancelloria eros constituye el primer registro para el Cámbrico de México.

\section{CONCLUSIONES}

El Cerro El Sahuaral constituye una nueva localidad fosilífera para el Cámbrico medio (Serie 3, Piso 5) de Sonora central. La sucesión mixta de calcilutita rojiza e intercalaciones de caliza de estratificación fina (mudstone) de la Formación El Gavilán, corresponde a un paleoambiente de plataforma abierta, somera y de aguas cálidas. Entre la fauna recolectada de chancellóridos destacan fragmentos de escleritomos de Chancelloria eros Walcott, 1920, y escleritos asignados 


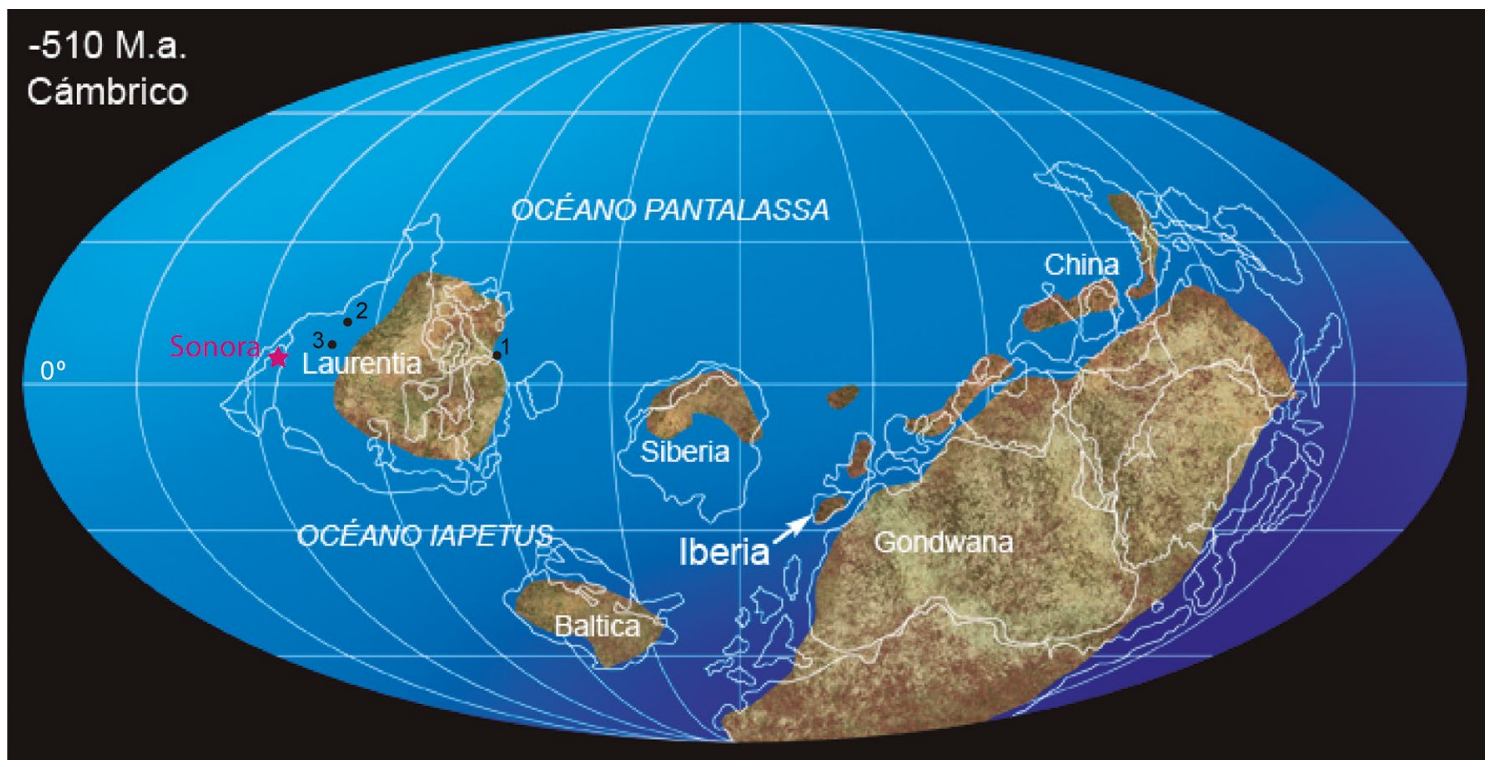

Figura 7. Palaeogeografía del Cámbrico con la ubicación de Sonora, México y de 1) Sirius Passet, Groenlandia. 2) Burgess Shale, Canadá. 3) Formación Marjum y Wheeler Shale, Utah, USA como parte de la plataforma de Laurentia (extraído del Instituto de Investigación en Ciencias Ambientales de Aragón, Universidad de Zaragoza, España y modificado). <http://iuca.unizar.es/sites/default/files/grupos/510Ma\%20Cambrico\%20paleogeografia.jpg $>$, acceso libre, consulta: 28 de Mayo de 2018.

a Allonnia tintinopsis Bengtson y Collins, 2015 los que se describen e ilustran por primera vez para el Cámbrico de México, así como escleritos asignados a Archiasterella sp., y numerosos rayos sueltos desarticulados de Chancelloria eros Walcott, 1920. Además, componen la fauna trilobites, braquiópodos liguliformes, esponjas reticulosas (hexactinéllidas), hiolítidos y eocrinoideos.

Con base en la asociación de trilobites (Tonkinella valida asociado a Quadragnostus sp. y Elrathina antiqua ) se determinó la Subzona de Altiocculus, parte superior de la Zona de Ehmaniella correspondiente a la Serie 3, Piso 5 (Cámbrico medio). El presente trabajo constituye el segundo registro de la Zona de Ehmaniella para México.

La fauna de chancellóridos de la Formación El Gavilán en la sección de El Sahuaral presenta afinidades con la biota de la misma formación en el Cerro El Chihuarruita de San José de Gracia, Sonora. A nivel continental, la fauna de Sonora compuesta por Chancelloria eros, Allonnia y Archisterella está presente en localidades de la plataforma cámbrica de Laurentia, específicamente en Burgess Shale y la Formación Marjum y Weeler Shale de Utah, EUA. La posición del continente de Laurentia cercana al paleoecuador durante el Cámbrico medio fue un factor favorable para el florecimiento de diversos grupos de organismos que se movilizaron a través de corrientes de la plataforma somera de aguas cálidas que rodeaba el continente. Las rocas cámbricas de Sonora formaron parte de esa plataforma durante el Cámbrico.

\section{AGRADECIMIENTOS}

Los autores agradecen el apoyo financiero brindado por el proyecto CONACYT No. 165826, 235970 y ECOS Francia-México No. M13-U01 y al CONICET de Argentina. Se agradece al M.C.J. Alfredo Ochoa (USON) la ayuda brindada en las tareas de campo y a Daniel Dueñas (Conicet, CCT-Mendoza) la confección de las figuras. Muy especialmente al Dr. Stefan Bengtson (Museo de Historia Natural de Estocolmo, Suecia) por los comentarios sobre los chancellóridos de Sonora. Las sugerencias realizadas por el editor y dos revisores anónimos ayudaron a mejorar este trabajo.

\section{REFERENCIAS}

Almazán-Vázquez, E., 1989, El Cámbrico-Ordovícico de Arivechi en la región centro-oriental del Estado de Sonora: Universidad Nacional Autónoma de México, Instituto de Geología, Revista, 8(1), 58-66.

Bengtson, S., 1990, Chancelloriids, in Bengtson, S., Conway Morris, S., Cooper, B.J., Jell, P.A., Runnegar, B.N. (eds.), Early Cambrian fossils from South Australia: Association of Australasian Palaeontologists Memory 9, 45-69.

Bengtson, S., 2000. Teasing fossils out of shales with cameras and computers: Palaeontologia Electronica, 3, 1.4, 1-14

Bengtson, S., Collins, D., 2015, Chancelloriids of the Cambrian Burgess Shale: Palaeontologia Electronica, 18, 1.6A, 1-67.

Bengtson, S., Hou, X.G., 2001, The integument of Cambrian chancelloriids: Acta Palaeontologica Polonica, 46, 1-22.

Bengtson, S., Missarzhevsky V.V., 1981, Coeloscleritophora a major group of enigmatic Cambrian metazoans. 19-21, en Taylor, M. E. (ed.), Short papers from the Second International Symposium on the Cambrian System: US Geological Survey Open-File Report, 81-743.

Beresi, M.S., Rigby, J.K., 1994, Sponges and Chancelloriids from the Cambrian of Western Argentina: Journal of Paleontology, 68(2), 208-217.

Beresi M.S., Rigby, J.K., 2013, Middle Cambrian protospongiid sponges and chancelloriids from the Precordillera of Mendoza Province, western Argentina: Neues Jahrbuch fur Geologie und Palaontologie Abhandlungen, 268, 259-274.

Beresi, M.S., Cuen-Romero, F., Montijo-González, A., Buitrón-Sánchez, B., Minjárez-Sosa, J., De la O Villanueva, M., Palafox-Reyes, J., 2013 , Chancelloriida Walcott, 1920 y Reticulosa Reid, 1958 (protospóngidos) del Cámbrico de San José de Gracia, Sonora, en VIII Congreso latinoamericano de Paleontología y XIII Congreso Nacional de Paleontología: Guanajuato, México, Resúmenes, p. 38.

Beresi, M.S., Botting, J. P., Palafox, J.J., Buitrón-Sánchez, B.E., 2017, New reticulosan sponges from the middle Cambrian of Sonora, Mexico: Acta Palaeontologica Polonica 62 (4), 691-703.

Buitrón, B.E., 1992, Las rocas sedimentarias marinas del Paleozoico inferior de México y su contenido biótico, en Gutiérrez-Marco, J.G., Saavedra, J., Rábano, I. (eds), Paleozoico inferior de Ibero-América: Universidad de Extremadura, UNESCO, IGCP, Proyectos,193- 201

Buitrón, B.E., Vachard, D., Clausen S., Gómez, E.C., 2011, Moluscos del Cámbrico (Hyolithida, Syssoiev, 1957) de San José de Gracia, Sonora, Noroeste de México, en Félix-Pico, E.F., Bertsch, H., Campay, R.C. (eds.), 
Reunión Internacional de Malacología: La Paz BCS, México, Resumen, p.175.

Conway-Morris, S., Peel, J.S., Higgins, A.K., Soper, N.J., Davis, N.C. 1987, A Burgess shale-like fauna from the Lower Cambrian of North Greenland: Nature 326, 181-183.

Cooper, G.A., Arellano, A.R.V., Johnson, J.H., Okulitch, V.J., Stoyanow, A., Lochman, C., 1952, Cambrian Stratigraphy and Paleontology near Caborca, northwestern Sonora, México: Smithsonian Miscellaneous Collections, 119, 1-184.

Cooper, G.A., Arellano, A.R.V., Johnson, J.H., Okulitch, V.J., Stoyanow, A., Lochman, C., 1956, Geología y Paleontología de la región de Caborca, norponiente de Sonora: Pt. 1 ${ }^{\text {a: }}$ Universidad Nacional Autónoma de México, 259 pp.

Cuen, F.J., 2012, Sobre la Estratigrafía y Paleontología del Cámbrico del área de San José de Gracia, Sonora central: Sonora, México, Universidad de Sonora, Departamento de Geología, Tesis de Licenciatura, 73 pp.

Cuen, F.J., 2013, Bioestratigrafía del Cámbrico de San José de Gracia, Sonora. Consideraciones Paleogeográficas: Sonora, México, Universidad de Sonora, Departamento de Geología, Tesis de Maestría en CienciasGeología, $142 \mathrm{pp}$.

Cuen, F.J., Beresi, M.S., Montijo, A., Buitrón, B.E., Minjárez, I., De la O, M., Palafox, J.J., 2013, Chancelloriia Walcott, 1920 y Reticulosa Reid, 1958 del Cámbrico medio de San José de Gracia, Sonora, México: Boletín de la Sociedad Geológica Mexicana, 65(3), 581-590.

Cuen-Romero, F.J., Valdez-Holguín, J.E., Buitrón-Sánchez, B.E., Monreal, R., Sundberg, F., Montijo-González, A., Minjárez-Sosa, I., 2016, Cambrian Stratigraphy of San José de Gracia, Sonora, México: El Gavilán Formation, a new lithostragraphic unit of middle Cambrian open shelf environment: Boletín de la Sociedad Geológica Mexicana, 68(3), 429-441.

Cuen-Romero, F.J., Valdez-Holguín J.E., Buitrón-Sánchez, B.E., Monreal, R., Enríquez-Ocaña, L.F., Aguirre-Hinojosa, E., Ochoa-Granillo, J.A., PalafoxReyes J.J., 2018, Trilobite-based biostratigraphy (arthropoda-trilobita) and related faunas of the Cambrian from Sonora, Mexico: Journal of South American Earth Sciences, 83, 227-236.

Doré, F., Reid, R. E., 1965, Allonnia tripodophora nov. gen., nov. sp., nouvelle Eponge du Cambrien inferieur de Carteret (Manche): Compte Rendu Sommaire des Sciences de la Societe Geologique de France, 1965, 20-21.

Fernández-Remolar, D.C., 2001, Chancelloridae del Ovetiense Inferior de la Sierra de Córdoba, España: Revista Española de Paleontología, 16, 39-61.

González-León, C., 1986, Estratigrafía del Paleozoico de la Sierra del Tule, noreste de Sonora: Revista del Instituto de Geología, Universidad Nacional Autónoma de México, 6, 117-135.

Hong, J., Choh, S.J., Lee, D.-J., 2016, Distribution of Chancelloriids in a Middle Cambrian carbonate platform deposits, Taebaek Group, Korea: Acta Geologica Sinica, 90 (3),783-795.

Janusen, D., Sateiner, M., Maoyan, Z., 2002, New well preserved scleritomes of Chancelloridae from the Early Cambrian Yuanshan Formation (Chengjiang, China) and the Middle Cambrian Wheeler Shale (Utah, USA) and Paleobiological Implications: Journal of Paleontology, 76(4), 596-606.

Lochman, C., 1948, New Cambrian trilobite genera from northwest Sonora, Mexico: Journal of Paleontology, 22, 451-464.

McMenamin, M.A.S., 2008, Early Cambrian sponge spicules from the Cerro San Clemente Caborca, Sonora, Mexico: Acta Geológica, 6(4), 363-367.
Mehl, D., 1996, Organization and microstructure of the chancelloriid skeleton: implications for the biomineralization of the Chancelloriidae: Bulletin de l'Institut Oceanographique de Monaco, $\mathrm{N}^{\circ}$ Special, 14(4), 377-385.

Mehl, D., 1998, Porifera and Chancelloriidae from the Middle Cambrian of the Georgina Basin, Australia: Palaeontology, 41, 1153-1182.

Moore, J.L., Guoxiang, LI, G., Porter, S.M., 2014, Chancelloriid sclerites from the lower Cambrian (Meishucunian) of eastern Yunnan, China, and the early history of the group: Palaeontology, 57(4), 833-878.

Nardin, E., Almazán-Vásquez, E., Buitrón-Sánchez, B.E., 2009, First report of Gogia (Eocrinoidea-Echinodermata) from the early-middle Cambrian of Sonora (Mexico), with biostratigraphical and palaeoecological comments: Geobios 42, 233-242.

Qian, Y., Bengtson, S., 1989, Palaeontology and biostratigraphy of the Early Cambrian Meishucunian Stage in Yunnan Province, South China: Fossils and Strata, 24, $156 \mathrm{pp}$.

Randell, R.D., Lieberman, B.S., Hasiotis, S.T., Pope, M.C., 2005, New chancelloriids from the Early Cambrian Sekwi Formation with a comment on chancelloriid affinities: Journal of Paleontology, 79, 987-996.

Rigby, J.K., 1978, Porifera of the Middle Cambrian Wheeler Shale, from the Wheeler Amphitheater, House Range, in western Utah: Journal of Paleontology, 52, 1325-1345.

Sdzuy, K., 1969, Unter- und mittelkambrische Porifera (Chancelloriida und Hexactinellida): Palaontologische Zeitschrift, 43, 115-147.

Stewart, J.H., Poole, F.G., 2002, Inventory of Neoproterozoic and Paleozoic strata in Sonora, Mexico: United States Geological Survey Open-File Report, 02-97, 1-50.

Sundberg, F.A., 1994, Corynexochida and Ptychopariida (Trilobita, Arthropoda) of the Ehmaniella Biozone (Middle Cambrian), Utah and Nevada: Contributions in Science, Natural History Museum of Los Angeles, 446, $137 \mathrm{pp}$.

Vega-Granillo, R., 1996, Geología de la Sierra de Mazatán: Boletín del Departamento de Geología, Universidad de Sonora, 13(1), 89-102.

Walcott, C.D., 1920, Middle Cambrian Spongiae: Smithsonian Miscellaneous Collections, 67(6), 261-364.

Yun H., Zhang, X., Li, L., 2017, Chancelloriid Allonnia erjiensis sp. nov. from the Chengjiang Lagerstätte of South China: Journal of Systematic Palaeontology, 16(5), 435-444. https://doi.org/10.1080/14772019.2017. 1311380.

Zhang, X.L., Liu, W., Zhao, Y.-L., 2008, Cambrian Burgess Shale-type Lagerstätten in South China: distribution and significance: Gondwana Research, 14, 255-262.

Zhao, Y.L., Yuan, J.L., Zhu, M.Y., Yang, R.D., Qian, Y., Huang, Y.Z., Guo, Q.J., Pan, Y., 1999, A progress report on research on the early Middle Cambrian Kaili biota, Guizhou Province, PRC: Acta Palaeontologica Sinica, 38, (in Chinese with English abstract), supplement, 1-14.

Manuscrito recibido: junio 6, 2018

Manuscrito corregido recibido: octubre 16, 2018

Manuscrito aceptado: octubre 16, 2018 